\title{
Numerical Solution for Lotka-Volterra Model of Oscillating Chemical Reactions with Interactive Fuzzy Initial Conditions
}

\author{
Vinícius F. Wasques ${ }^{a}$, Estevão Esmi ${ }^{b}$, Laécio C. Barros ${ }^{c}$ and P. Sussner ${ }^{d}$ \\ ${ }^{a}$ Institute of Mathematics, Statistics and Scientific Computing, Unicamp, Brazil, vwasques@outlook.com \\ ${ }^{2}$ Institute of Mathematics, Statistics and Scientific Computing, Unicamp, Brazil, eelaureano@gmail.com \\ ${ }^{3}$ Institute of Mathematics, Statistics and Scientific Computing, Unicamp, Brazil, laeciocb@ime.unicamp.br \\ ${ }^{4}$ Institute of Mathematics, Statistics and Scientific Computing, Unicamp, Brazil, sussner@ime.unicamp.br
}

\begin{abstract}
In this paper we study Lotka-Volterra model of oscillating chemical reactions via fuzzy differential equations, where the initial conditions are given by interactive fuzzy numbers. The concept of interactivity is associated with the notion of joint possibility distribution. The fuzzy solution is given by a numerical method which considers fuzzy arithmetic with interactivity. We present some examples to illustrate that different types of interactivity produce different solutions.
\end{abstract}

Keywords: Fuzzy initial value problem, Sup- $J$ extension principle, Interactive fuzzy numbers, Chemical reactions.

\section{Introduction}

Chemical kinetic deals with chemistry experiments and interprets them in terms of mathematical models. In particular, chemical kinetics studies the chemical reactions, as well as the factors that influence the final result [7]. We focus on chemical reactions of the type $U+V \rightarrow c W$, where $U$ and $V$ are the consumed reagents and $W$ is the final product of this reaction, with proportion $c$.

Some factors may influence the velocity of these reactions, for instance, concentration, activation energy, temperature, pressure etc. The velocity $(v)$ of a reaction can be determined from $v=k[U]^{m}[V]^{n}$, where $k$ is the reaction rate, $[U]$ and $[V]$ are the concentration of the reagents and $m$ and $n$ are the orders of the reactions, which are determined experimentally. Thus, there may be imprecision (or uncertainty) in the process of obtaining such parameters. The classic models do not consider this fact [9]. On the other hand, fuzzy sets theory can be used to describe these uncertainties.

In this manuscript we focus on Lotka-Volterra model of oscillating chemical reactions, which is based on a molecular mechanism where at each step the reagent molecules combine to produce intermediate reagents or final products. Fundamentally we have [13]:

$$
\begin{aligned}
& A+X \rightarrow 2 X \text { with reaction rate } k_{1} \\
& X+Y \rightarrow 2 Y \text { with reaction rate } k_{2} \\
& Y \rightarrow B \text { with reaction rate } k_{3}
\end{aligned}
$$

The effective rate laws for the reagent $A$, the product $B$, and the intermediates reagents $X$ and $Y$ are described by the initial value problem (IVP) [10]:

$$
\left\{\begin{array}{lll}
\frac{d[A]}{d t}=-k_{1}[A][X], & & {[A(0)]=\left[A_{0}\right]} \\
\frac{d[X]}{d t}=k_{1}[A][X]-k_{2}[X][Y], & {[X(0)]=\left[X_{0}\right]} \\
\frac{d[Y]}{d t}=k_{2}[X][Y]-k_{3}[Y], & & {[Y(0)]=\left[Y_{0}\right]} \\
\frac{d[B]}{d t}=k_{3}[Y], & & {[B(0)]=\left[B_{0}\right]}
\end{array} .\right.
$$

Note that

$$
[A(t)]+[X(t)]+[Y(t)]+[B(t)]=k, \quad \forall t \in \mathbb{R}
$$

for some $k \in \mathbb{R}$, since

$$
\frac{d[A]}{d t}+\frac{d[X]}{d t}+\frac{d[Y]}{d t}+\frac{d[B]}{d t}=0 .
$$

In particular

$$
\left[A_{0}\right]+\left[X_{0}\right]+\left[Y_{0}\right]+\left[B_{0}\right]=k .
$$

The initial conditions and/or parameters may be uncertain [9]. In case where the initial conditions $\left[A_{0}\right]$, $\left[X_{0}\right],\left[Y_{0}\right]$ and $\left[B_{0}\right]$ are uncertain and modeled by fuzzy numbers, we have that $\left[A_{0}\right],\left[X_{0}\right],\left[Y_{0}\right]$ and $\left[B_{0}\right]$ need to be interactive $[8]$ in order to guarantee that the total quantity $(k)$, given in (1.4), be a real number [2].

Interactivity is a relationship between fuzzy numbers that resembles the concept of dependence in the case 
of random variables. In this paper we use this concept to ensure that Equation (1.4) holds. Moreover, we use interactivity to model the intrinsic dependence of the reagents/products with its concentration [6].

The IVP whose initial conditions are given by fuzzy numbers is called by fuzzy initial value problem (FIVP).

In order to provide a numerical solution for this FIVP, we use the method proposed in [16]. This method can be used for any n-dimensional system of differential equations and it consists in adapting the arithmetic operations of the classical Runge-Kutta method of order 1 (Euler), by means of sup- $J$ extension principle, for the arithmetic between interactive fuzzy numbers.

Finally in order to verify the dependence of the final result with the concetration factors, we present some examples to illustrate that different types of interactivity result in different solutions for System (1.2).

\section{Mathematical background}

In this section we present the Euler's method and some basic concepts of fuzzy sets theory.

\subsection{Euler's method}

Let $y_{i}: \mathbb{R} \rightarrow \mathbb{R}^{n}$, with $i=1, \ldots, n$, be functions that depend on time $t$. Consider the ordinary differential equation (ODE) with initial value given by (2.5)

$$
\left\{\begin{array}{l}
\frac{d y_{i}}{d t}=f_{i}\left(t, y_{1}, y_{2}, \ldots, y_{n}\right) \\
y\left(t_{0}\right)=y_{0} \in \mathbb{R}^{n}
\end{array} \quad, i=1, \ldots, n\right.
$$

where $f_{i}: \mathbb{R}^{n+1} \rightarrow \mathbb{R}$ depends on $y_{1}, y_{2}, \ldots, y_{n}$ and $t$.

Euler's method consists in determining numerical solutions for ODEs described by (2.5). The algorithm is provided as follows

$$
y_{i}^{k+1}=y_{i}^{k}+h f_{i}\left(t_{k}, y_{1}^{k}, \ldots, y_{n}^{k}\right),
$$

with $0 \leq k \leq N-1$, where $N$ is the number of partitions that the interval is divided, $h$ is the size of the interval $\left[t_{k}, t_{k+1}\right]$ and $\left(t_{0}, y_{i}^{0}\right)$ is the initial condition.

\section{$2.2 \quad$ Fuzzy sets theory}

A fuzzy subset $A$ of a universe $X$ is associated with a function $\mu_{A}: X \rightarrow[0,1]$ called membership function, where $\mu_{A}(x)$ represents the membership degree of $x$ in $A$ for all $x \in X$. For notational convenience, we may simply use the symbol $A(x)$ instead of $\mu_{A}(x)$. The class of fuzzy subsets of $X$ is denoted by $\mathcal{F}(X)$. From now on, we assume that $X$ is a topological space.
The $\alpha$-cut of a fuzzy set $A \subseteq X$, denoted by $[A]^{\alpha}$, is defined by $[A]^{\alpha}=\{x \in X: A(x) \geq \alpha\}, \forall \alpha \in(0,1]$ and $[A]^{0}=\operatorname{cl}\{x \in X: A(x)>0\}$, where cl Y, $Y \subseteq X$, denotes the closure of $\mathrm{Y}[1]$.

A fuzzy set $\mathrm{A}$ of $\mathbb{R}$ is called a fuzzy number if all $\alpha$-cuts are bounded, closed and non-empty nested intervals for all $\alpha \in[0,1]$. Thus the $\alpha$-cuts of the fuzzy number $A$ are denoted by $[A]^{\alpha}=\left[a_{\alpha}^{-}, a_{\alpha}^{+}\right]$. The class of fuzzy numbers, denoted by $\mathbb{R}_{\mathcal{F}}$, represents a special class of fuzzy sets of $\mathbb{R}$ that includes the sets of the real numbers as well as the set of the bounded closed intervals of $\mathbb{R}$. In addition, we define the subclass $\mathbb{R}_{\mathcal{F}_{\mathcal{C}}}$ by the set of all fuzzy numbers such that the endpoints of its $\alpha$-cuts are continuous with respect to $\alpha$. The triangular fuzzy number is an example of element in $\mathbb{R}_{\mathcal{F}_{\mathcal{C}}}$. Recall that a triangular fuzzy number $A$ is denoted by the triple $(a ; b ; c)$ for some $a \leq b \leq c$. By means of $\alpha$-cuts we have $[A]^{\alpha}=[a+\alpha(b-a), c-\alpha(c-b)], \forall \alpha \in[0,1]$.

The width of a fuzzy number is defined by

$$
\operatorname{width}(A)=a_{0}^{+}-a_{0}^{-}, \quad \forall A \in \mathbb{R}_{\mathcal{F}}
$$

and it is associated with the uncertainty that the fuzzy number $A$ models.

A fuzzy relation $J \in \mathcal{F}\left(\mathbb{R}^{n}\right)$ is said to be a joint possibility distribution (JPD) among the fuzzy numbers $A_{1}, \ldots, A_{n} \in \mathbb{R}_{\mathcal{F}}$, if

$$
A_{i}(y)=\sup _{\left(x_{1}, \ldots, x_{n}\right): x_{i}=y} J\left(x_{1}, \ldots, x_{n}\right), \forall y \in \mathbb{R},
$$

for all $i=1, \ldots, n$.

For the special case that $J$ is given by

$$
J_{\wedge}\left(x_{1}, \ldots, x_{n}\right)=A_{1}\left(x_{1}\right) \wedge \ldots \wedge A_{n}\left(x_{n}\right),
$$

where $\wedge$ represents the minimum operator, the fuzzy numbers $A_{1}, \ldots, A_{n}$ are said to be non-interactive. Otherwise, that is when $J \neq J_{\wedge}$, the fuzzy numbers $A_{1}, \ldots, A_{n}$ are called $J$-interactive or simply interactive. Thus, the interactivity of the fuzzy numbers $A_{1}, \ldots, A_{n}$ arises from a given joint possibility distribution and this definition resembles the concept of dependence in the case of random variables.

One example of interactivity is the one based on the concept of linear interactivity (or complete correlation). This concept was introduced by Fullér et al. [3,8] for two fuzzy numbers. Subsequently, the authors of $[11,15]$ proposed an extension of this notion for $n$ fuzzy numbers, $n>2$. The fuzzy numbers $A_{1}, \ldots, A_{n}$ are said to be linearly interactive if there exist a joint possibility distribution $J=J_{L}$ given by

$$
J_{L}\left(x_{1}, \ldots, x_{n}\right)=A_{i}\left(x_{i}\right) \chi_{L}\left(x_{1}, \ldots, x_{n}\right),
$$


for all $\left(x_{1}, \ldots, x_{n}\right) \in \mathbb{R}^{n}$ and $i=1, \ldots, n$, where $\chi_{L}$ stands for the characteristic function of the set $L=$ $\left\{\left(u, q_{2} u+r_{2}, \ldots, q_{n} u+r_{n}\right): \forall u \in \mathbb{R}\right\}$.

The JPD $J_{L}$ can be used in several problems, for example, in fuzzy differential equations [2, 11, 15] and least squared methods [12]. However this JPD can only be applied to fuzzy numbers that have a co-linear relationship among their membership functions, which means that it can not be used for fuzzy numbers that do not have the same shape.

Next we present a more general joint possibility distribution that can be applied to every pair of fuzzy numbers.

Given $A_{1}, A_{2} \in \mathbb{R}_{\mathcal{F}_{\mathcal{C}}}$, for each $z \in \mathbb{R}$ and $\alpha \in[0,1]$ consider the auxiliary functions $g_{\wedge}^{i}, g_{\vee}^{i}$ and $v^{i}$ defined by $[5]$

$$
\begin{aligned}
& g_{\wedge}^{i}(z, \alpha)=\bigwedge_{w \in\left[A_{3-i}\right]^{\alpha}}|w+z|, \\
& g_{\vee}^{i}(z, \alpha)=\bigvee_{w \in\left[A_{3-i}\right]^{\alpha}}|w+z|
\end{aligned}
$$

and

$$
v^{i}(z, \alpha, \gamma)=(1-\gamma) g_{\wedge}^{i}(z, \alpha)+\gamma g_{\vee}^{i}(z, \alpha),
$$

for all $z \in \mathbb{R}, \alpha \in[0,1], \gamma \in[0,1]$ and $i \in\{1,2\}$.

Consider the sets $R_{\alpha}^{i}$ and $L^{i}(z, \alpha, \gamma)$ given by

$$
R_{\alpha}^{i}=\left\{\begin{array}{cl}
\left\{a_{i_{\alpha}}^{-}, a_{i_{\alpha}}^{+}\right\} & \text {if } \alpha \in[0,1) \\
{\left[A_{i}\right]^{1}} & \text { if } \alpha=1
\end{array}\right.
$$

and

$$
L^{i}(z, \alpha, \gamma)=\left[A_{3-i}\right]^{\alpha} \cap[l, r]
$$

where $l=-v^{i}(z, \alpha, \gamma)-z$ and $r=v^{i}(z, \alpha, \gamma)-z$.

Finally, we define $J_{\gamma}$ by

$$
J_{\gamma}\left(x_{1}, x_{2}\right)=\left\{\begin{array}{cl}
A_{1}\left(x_{1}\right) \wedge A_{2}\left(x_{2}\right), & \text { if }\left(x_{1}, x_{2}\right) \in P(\gamma) \\
0, & \text { otherwise }
\end{array}\right.
$$

with

$$
P(\gamma)=\bigcup_{i=1}^{2} \bigcup_{\alpha \in[0,1]} W_{i, \alpha}
$$

where $W_{i, \alpha}=\left\{\left(x_{1}, x_{2}\right): x_{i} \in R_{\alpha}^{i}, x_{3-i} \in L^{i}\left(x_{i}, \alpha, \gamma\right)\right\}$.

Esmi et al. [5] proved that $J_{\gamma}$, given by (2.10), is a joint possibility distribution of $A_{1}$ and $A_{2}$ for all $\gamma \in$ $[0,1]$. Intrinsically the parameter $\gamma$ models the "level" of interactivity between the fuzzy numbers $A_{1}$ and $A_{2}$, in the following sense, the closer of 0 the value of $\gamma$, the higher is the interactivity.
Recall that for $\gamma=1$ we have $J_{1}=J_{\wedge}($ see $(2.8))$. This means that if the chosen JPD is $J_{1}$, then we are dealing with non-interactive fuzzy numbers [5]. On the other hand for $\gamma=0$ we have that $J_{0}$ resembles $J_{L}(\operatorname{see}(2.9))[16]$.

The following definition is a generalization of the Zadeh's extension principle [17], which is used to extend classical functions to functions with fuzzy numbers as arguments.

Definition 1. [8] Let $J \in \mathcal{F}\left(\mathbb{R}^{n}\right)$ be a joint possibility distribution of $\left(A_{1}, \ldots, A_{n}\right) \in \mathbb{R}_{\mathcal{F}}^{n}$ and $f: \mathbb{R}^{n} \rightarrow \mathbb{R}$. The sup- $J$ extension of $f$ at $\left(A_{1}, \ldots, A_{n}\right) \in \mathbb{R}_{\mathcal{F}}^{n}$, denoted by $f_{J}\left(A_{1}, \ldots, A_{n}\right)$, is the fuzzy set given by:

$$
f_{J}\left(A_{1}, \ldots, A_{n}\right)(y)=\sup _{\left(x_{1}, \ldots, x_{n}\right) \in f^{-1}(y)} J\left(x_{1}, \ldots, x_{n}\right),
$$

where $f^{-1}(y)=\left\{\left(x_{1}, \ldots, x_{n}\right) \in \mathbb{R}^{n}: f\left(x_{1}, \ldots, x_{n}\right)=y\right\}$ is the inverse image of the function $f$ at $y$.

From Definition 1 is possible to establish an arithmetic on interactive fuzzy numbers. For example, the interactive sum between $A_{1}$ and $A_{2}$ is defined by:

$$
\left(A_{1}+{ }_{J} A_{2}\right)(y)=\sup _{x_{1}+x_{2}=y} J\left(x_{1}, x_{2}\right),
$$

where $f\left(x_{1}, x_{2}\right)=x_{1}+x_{2}$ and $J$ is an arbitrary JPD of $A_{1}$ and $A_{2}$.

The method proposed in [16] considers the joint possibility distributions $J_{\gamma}$ between translated fuzzy numbers, in order to control the width of the numerical solutions [14]. Here we use the same approach. For simplicity of notation we denote the arithmetic operations $A_{1} \otimes_{J_{\gamma}} A_{2}$ by $A_{1} \otimes_{\gamma} A_{2}$, where $\otimes \in\{+,-, \cdot, \div\}$.

In order to illustrate these arithmetic operations, let us provide an example of the interactive addition $+_{\gamma}$ between fuzzy numbers.

Example 1. Let $A_{1}=A_{2}=(-1 ; 0 ; 1) \in \mathbb{R}_{\mathcal{F}_{\mathcal{C}}}$. For $\gamma \in\{0,0.5,0.75,1\}$, we have

$$
\begin{aligned}
A_{1}+{ }_{0} A_{2} & =0 \\
A_{1}+{ }_{0.5} A_{2} & =(-1 ; 0 ; 1) \\
A_{1}+{ }_{0.75} A_{2} & =(-1.5 ; 0 ; 1.5) \\
A_{1}+{ }_{1} A_{2} & =(-2 ; 0 ; 2) .
\end{aligned}
$$

Note that in Example 1 we obtain $A_{1}+{ }_{0} A_{2}=0$, where 0 stands for the fuzzy number 0 whose membership function is given by the characteristic function $\chi_{\{0\}}$. This result arises from the fact that $A_{2}=-A_{1}$.

In addition, for $\gamma=1$ we have $A_{1}+{ }_{1} A_{2}=(-2 ; 0 ; 2)=$ $A_{1}+A_{2}$, where the symbol " + " represents the standard sum on fuzzy numbers, corroborating the previous observation. 
Observe that

$$
\begin{aligned}
\text { width }\left(A_{1}+{ }_{0} A_{2}\right) & \subseteq \operatorname{width}\left(A_{1}+{ }_{0.5} A_{2}\right) \\
& \subseteq \operatorname{width}\left(A_{1}+{ }_{0.75} A_{2}\right) \\
& \subseteq \operatorname{width}\left(A_{1}+{ }_{1} A_{2}\right) .
\end{aligned}
$$

This statement holds true, since the width of the interactive sum via $J_{\gamma}$ is increasing with respect to the parameter $\gamma \in[0,1][5,14]$.

In the next section we provide the numerical solutions for (1.2), based on this methodology.

\section{Fuzzy numerical solution}

In this paper we consider that the initial conditions $\left[A_{0}\right],\left[X_{0}\right],\left[Y_{0}\right]$ and $\left[B_{0}\right]$ are given by interactive fuzzy numbers. Hence the sum given by Equations (1.3) and (1.4) must be adapted for these type of fuzzy numbers.

We use the sum obtained via sup- $J$ extension principle, with $J=J_{\gamma}$ (see (2.7)) defined in the previous section. Consequently the sum operation depends on the values of $\gamma \in[0,1]$. Thus Equations (1.3) and (1.4) become

$$
[A(t)]+{ }_{\gamma}[X(t)]+_{\gamma}[Y(t)]+_{\gamma}[B(t)]=k,
$$

and

$$
\left[A_{0}\right]+{ }_{\gamma}\left[X_{0}\right]+{ }_{\gamma}\left[Y_{0}\right]+{ }_{\gamma}\left[B_{0}\right]=k \text {. }
$$

Since $[B]$ represents the concentration of the final product $B$, we have $\left[B_{0}\right]=0$. The combination of (3.14) and (3.13) leads us to the following

$[B]=\left[A_{0}\right]-_{\gamma}[A(t)]+_{\gamma}\left[X_{0}\right]-_{\gamma}[X(t)]+_{\gamma}\left[Y_{0}\right]-_{\gamma}[Y(t)]$

Therefore it is only necessary to solve the first three equations of (1.2).

The numerical solution for this problem is based on the classical Euler's method extending the arithmetic operations to arithmetic operations for interactive fuzzy numbers, as described in Subsection 2.2. Hence the fuzzy numerical solution is given by

$$
\left\{\begin{array}{l}
{[A]^{k+1}=[A]^{k}-_{\gamma} h k_{1}\left([A]^{k} \cdot{ }_{\gamma}[X]^{k}\right)} \\
{[X]^{k+1}=[X]^{k}+_{\gamma} h\left(k_{1}[A]^{k} \cdot \gamma[X]^{k}-_{\gamma} k_{2}[X]^{k} \cdot_{\gamma}[Y]^{k}\right)} \\
{[Y]^{k+1}=[Y]^{k}+_{\gamma} h\left(\left(k_{2}[X]^{k} \cdot{ }_{\gamma}[Y]^{k}\right)-_{\gamma}\left(k_{3}[Y]^{k}\right)\right)} \\
{[B]^{k+1}=\left[A_{0}\right]-_{\gamma}[A]^{k}+_{\gamma}\left[X_{0}\right]-_{\gamma}[X]^{k}+_{\gamma}\left[Y_{0}\right]-_{\gamma}[Y]^{k}}
\end{array}\right.
$$

with initial conditions $\left[A_{0}\right],\left[X_{0}\right],\left[Y_{0}\right] \in \mathbb{R}_{\mathcal{F}}$.

Figures 1, 2 and 3 depict the simulations for three different "levels" of interactivity, that is, $\gamma=0, \gamma=$ 0.5 and $\gamma=0.75$. The parameters used were $h=$
A
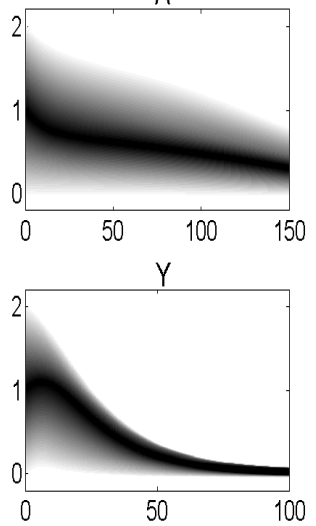

$X$

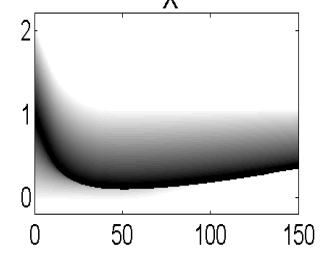

B

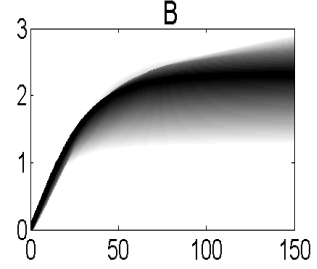

Figure 1: Numerical solution for $\gamma=0$. The gray lines represent the $\alpha$-cuts of the fuzzy solutions, where their endpoints for $\alpha$ varying from 0 to 1 are represented respectively from the gray-scale lines varying from white to black.
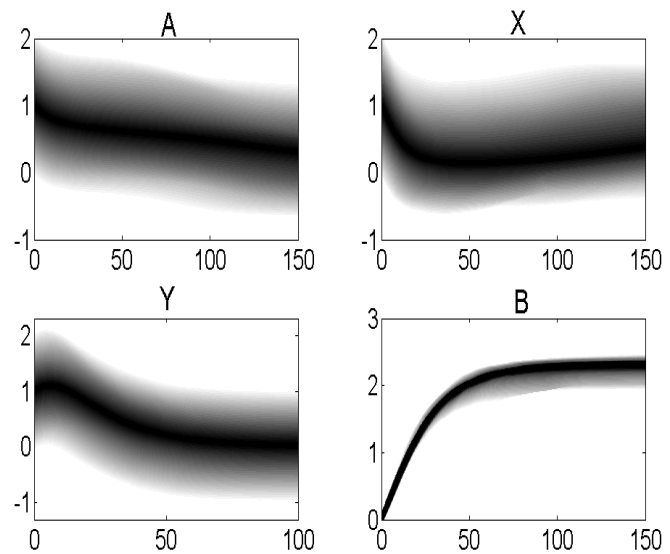

Figure 2: Numerical solution for $\gamma=0.5$. The gray lines represent the $\alpha$-cuts of the fuzzy solutions, where their endpoints for $\alpha$ varying from 0 to 1 are represented respectively from the gray-scale lines varying from white to black.

$0.125, k_{1}=0.03, k_{2}=0.09, k_{3}=0.06$ and $\left[A_{0}\right]=$ $\left[X_{0}\right]=\left[Y_{0}\right]=(0 ; 1 ; 2)$.

Note that for different values of $\gamma$ we obtain different final products. This fact is associated with the interactive arithmetic that is based on the family of the joint possibility distribution $J_{\gamma}[16]$.

In Figure 1 we observe that for the highest level of interactivity $(\gamma=0)$ we obtain decreasing width for the reagents $A, X$ and $Y$ over time. However the width of the final product increases initially and thereafter has few variations. 

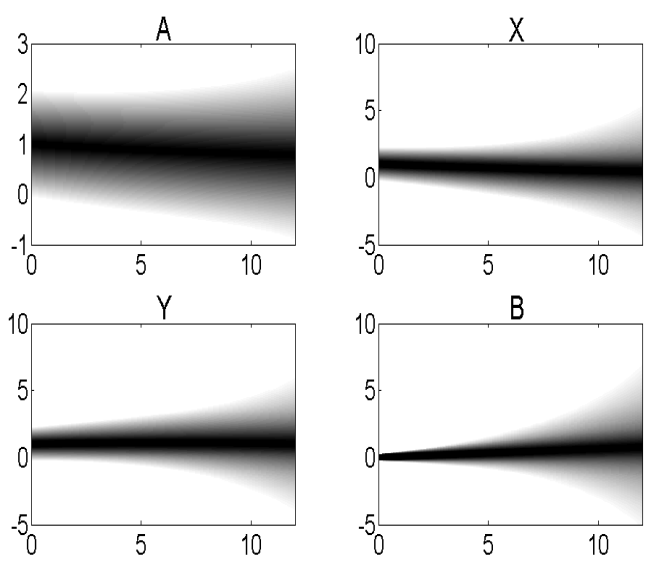

Figure 3: Numerical solution for $\gamma=0.75$. The gray lines represent the $\alpha$-cuts of the fuzzy solutions, where their endpoints for $\alpha$ varying from 0 to 1 are represented respectively from the gray-scale lines varying from white to black.

Figure 2 reveals that for $\gamma=0.5$ (medium level of interactivity) the width of $A, X$ and $Y$ has few variations. The width of the product also has few variations but always with width smaller than width of the fuzzy solution provided by $\gamma=0$.

Even though for $\gamma=0.5$ the reagents have a greater uncertainty than for $\gamma=0$, we have that the uncertainty in the final product is smaller. Thus, in this sense, the solution via $J_{0.5}$ may describe this final product in a more precisely way.

For $\gamma=0.75$ we expect that the uncertainty increases over time, since the value of $\gamma$ is closer to 1 [16]. This fact is corroborated in Figure 3.

Figure 4 illustrates the deterministic solution of the system $(1.2)$ with initial values $\left[a_{0}\right]=\left[x_{0}\right]=\left[y_{0}\right]=1$.

From the chemical point of view, the joint possibility distributions $J_{0}$ and $J_{0.5}$ produce solutions which are qualitatively similar to the deterministic case (see Figures 1, 2 and 4). On the other hand, the joint possibility distribution $J_{0.75}$ produces a numerical solution with uncertainty so high that the final result does not resemble (qualitatively) the deterministic case.

Hence we verify that, in the context of fuzzy sets theory, the relationship of interactivity (as well as the level of interactivity given by $\gamma$ ) influences in the final product. This means that from the chemical point of view, different quantities and/or concentration of the reagents produce products with different uncertainties.
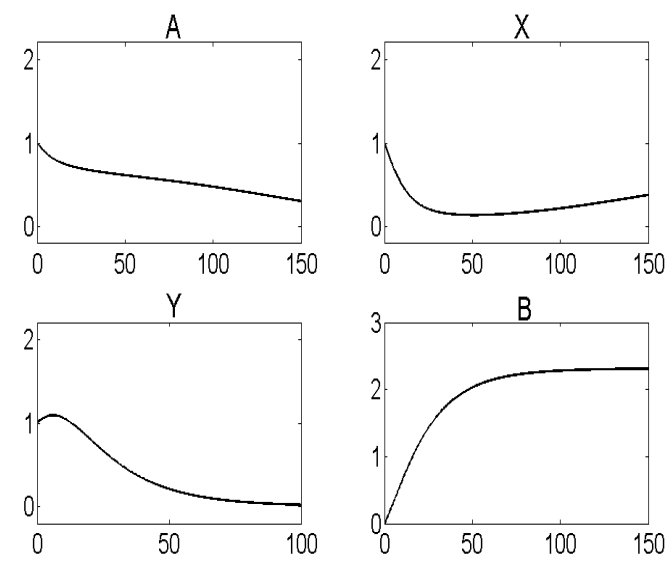

Figure 4: The deterministic numerical solution.

\section{Final remarks}

In this work we studied the Lotka-Volterra model of oscillating chemical reactions from the point of view of fuzzy sets theory. More precisely, in order to take into account possible uncertainties in the concentration of the reagents, we provided a numerical solution for this model considering that the initial conditions are given by interactive fuzzy numbers.

The relationship of interactivity models the dependence of the final product with the concentration of the initial and intermediary reagents. We verified that the interactivity interferes in the width of the fuzzy numerical solution, which describes the uncertainty of concentration of the reagents and the final product.

Differently of a fuzzy initial value problem where the derivative is obtained by a fuzzy process $[2,4]$, here we only used numerical methods for FIVPs provided in the literature, to observe how the relationship of interactivity acts in the chemical reagents when the initial conditions were given by interactive fuzzy numbers.

\section{Acknowledgement}

The authors would like to thank the support of CNPq under grants nos. 142414/2017-4, 306546/20175 and 313145/2017-2, FAPESP under grant nos. 2016/26040-7 and 2018/13657-1 and CAPES - Finance Code 001.

\section{References}

[1] L. C. Barros, R. C. Bassanezi, W. A. Lodwick, A First Course in Fuzzy Logic, Fuzzy Dynamical Systems, and Biomathematics, Springer Berlin Heidelberg, Berlin, 2017. 
[2] L. C. Barros, F. S. Pedro, Fuzzy differential equations with interactive derivative, Fuzzy Sets and Systems 309 (2017) 64-80.

[3] C. Carlsson, R. Fullér, P. Majlender, Additions of completely correlated fuzzy numbers, in: Fuzzy Systems Proceedings IEEE International Conference, Budapest, Hungary, 2004.

[4] E. Esmi, F. S. Pedro, L. C. de Barros, W. Lodwick, Fréchet derivative for linearly correlated fuzzy function, Information Sciences 435 (2018) $150-160$.

[5] E. Esmi, P. Sussner, G. B. D. Ignácio, L. C. Barros, A parametrized sum of fuzzy numbers with applications to fuzzy initial value problems, Fuzzy Sets and Systems 331 (2018) 85-104.

[6] P. Flowers, K. Theopold, R. Langley, W. R. Robinson, Chemistry, OpenStax, Texas, 2015.

[7] G. F. Froment, K. B. Bischoff, J. Wilde, Chemical Reactor Analysis and Design, Random House, New York, 1988.

[8] R. Fuller, P. Majlender, On interactive fuzzy numbers, Fuzzy Sets and Systems 143 (2004) 355369 .

[9] K. Ghosh, J. Schlipf, Formal modeling of a system of chemical reactions under uncertainty, Journal of Bioinformatics and Computational Biology 12 (5) (2014) 1-15.

[10] R. H. Hering, Oscillations in lotka-volterra systems of chemical reactions, Journal of Mathematical Chemistry 5 (2) (1990) 197-202.

[11] D. S. Ibanez, E. Esmi, L. C. Barros, Linear ordinary differential equations with linearly correlated boundary values, in: IEEE International Conference on Fuzzy Systems (FUZZ-IEEE), Rio de Janeiro, Brazil, 2018.

[12] N. J. B. Pinto, V. F. Wasques, E. Esmi, L. C. Barros, Least squares method with interactive fuzzy coefficient: Application on longitudinal data, Fuzzy Information Processing, Springer International Publishing, Cham (2018) 132-143.

[13] I. Prigogine, Introduction to Thermodynamics of Irreversible Processes, Interscience Publishers, California, 1968.

[14] P. Sussner, E. Esmi, L. C. Barros, Controling the width of the sum of interactive fuzzy numbers with applications to fuzzy initial value problems, in: Fuzzy Systems IEEE International Conference, Vancouver, Canada, 2016.
[15] V. F. Wasques, E. Esmi, L. C. Barros, F. S. Pedro, P. Sussner, Higher order initial value problem with interactive fuzzy conditions, in: IEEE International Conference on Fuzzy Systems (FUZZIEEE), Rio de Janeiro, Brazil, 2018.

[16] V. F. Wasques, E. Esmi, L. C. Barros, P. Sussner, Numerical solutions for bidimensional initial value problem with interactive fuzzy numbers, Fuzzy Information Processing, Springer International Publishing, Cham (2018) 84-95.

[17] L. A. Zadeh, Concept of a linguistic variable and its application to approximate reasoning - i, Information Sciences 8 (1975) 199-249. 